American Journal of Pharmaceutical Education 2016; 80 (9) Article S22.

\title{
CORRECTION
}

\section{Correction to 117th Annual Meeting of the American Association of Colleges of Pharmacy, Anaheim, California, July 23-27, 2016}

\begin{abstract}
Errors were made in the author lists for two abstracts that appeared in Volume 80, Issue 5, Article S2 of the Journal. Here are the corrected versions:

A 10-Station Multiple Mini-Interview (MMI) for Undergraduate Pharmacy Admission: Reflecting on Six Years of Experience. Andrea J. Cameron, Leslie Dan Faculty of Pharmacy, University of Toronto, Allan Choi, Leslie Dan Faculty of Pharmacy, University of Toronto, Jacqui Herbert, University Health Network, Xueqing Liao, Trillium Health Partners, Linda D. MacKeigan, Leslie
\end{abstract}

Dan Faculty of Pharmacy, University of Toronto, Komail Nadeem, Leslie Dan Faculty of Pharmacy, University of Toronto.

Analysis of the Validity of the Multiple MiniInterview for Predicting Performance in the Professional Program at the Leslie Dan Faculty of Pharmacy. Komail Nadeem, Leslie Dan Faculty of Pharmacy, University of Toronto, Linda D. MacKeigan, Leslie Dan Faculty of Pharmacy, University of Toronto, Andrea J. Cameron, Leslie Dan Faculty of Pharmacy, University of Toronto. 